\title{
VALOR PREDICTIVO DE LA HIPERGLICEMIA EN LA MORTALIDAD DE LOS PACIENTES CON TRAUMATISMO CRÁNEO ENCEFÁLICO
}

\section{PREDICTIVE VALUE OF HIPERGLICEMIA IN THE MORTALITY OF TEC PATIENTS}

\author{
César Quito Santos ${ }^{1}$, Carlos Salas Ruiz ${ }^{2}$
}

\section{RESUMEN}

El trabajo tiene como objetivo determinar si la hiperglicemia tiene valor predictivo significativo en la mortalidad de los pacientes con Traumatismo Cráneo Encefálico (TEC). Para ello se realizó un estudio observacional prospectivo longitudinal analítico, se seleccionaron 164 pacientes con TEC. Utilizando como punto de cohorte para hiperglicemia valores mayores a $160 \mathrm{mg} / \mathrm{dL}$. La tasa mortalidad de los pacientes con TEC en el presente estudio fue del 17,1\%; de los cuales el $82,1 \%$ presentaron hiperglicemia; mientras que en los pacientes que no fallecieron solo el $1,4 \%$ presento hiperglicemia. La mortalidad es mayor en pacientes que presentan hiperglicemia, evidenciándose una asociación altamente significativa entre estas 2 variables en estudio. El nivel promedio de glicemia en los pacientes fallecidos fue de 173,4 $\mathrm{mg} / \mathrm{dL}$, mientras que en los no fallecidos fue de $95,22 \mathrm{mg} / \mathrm{dL}$, evidenciándose una diferencia estadísticamente muy significativa entre los promedios de glicemia y la mortalidad de los pacientes con TEC. En conclusión la hiperglicemia, mayor de $160 \mathrm{mg} / \mathrm{dL}$, se asocia significativamente con la mortalidad de los pacientes con TEC; con un valor predictivo positivo de 0,92 y un valor predictivo negativo de 0,96 .

Palabras clave: Hiperglicemia, mortalidad, Traumatismo Cráneo Encefálico, valor predictivo

\section{ABSTRACT}

The work's aim is to determine wether hyperglicemia has a significative predictive value in the mortality of TEC patients. Fort it In this longitudinal prospective analitical study, we was chose 164 TEC patients. We asumed the value of hyperglicemia as $>160 \mathrm{mg} / \mathrm{dL}$. It was found a mortality rate of $17.1 \%$. From which $82,1 \%$ presented hyperglicemia, while among patients who survived only $1,4 \%$ presented hyperglicemia, the mortality is higher in patients who presented hyperglicemia, certifying a highly significative asociaton between these 2 variables. The average glicemia level in died patients was $173,4 \mathrm{mg} / \mathrm{dL}$, while the ones who survived was $95,22 \mathrm{mg} / \mathrm{dL}$, evidencing a very significative statistical difference betwen the average of blood glucose level and the mortality of TEC patients. In Conclusion the hiperglicemia, higher than $160 \mathrm{mg} / \mathrm{dL}$ is significantly associated to mortality of TEC patients with a positive predictive value of 0,92 and a negative predictive of 0,92 .

Key words: Mortality, hiperglicemia, TEC, predictive value.

\section{INTRODUCCIÓN}

El traumatismo cráneo encefálico (TEC), es una patología frecuente en los servicios de emergencias, el mismo, que debido a su gravedad tiene repercusiones que pueden ser dramáticas en sus consecuencias, si no existe un manejo adecuado y oportuno para mantener la homeostasis del paciente el desenlace puede llegar a ser fatal $(1,2)$. La identificación de la gravedad del traumatismo es importante, ya que de ello dependerá la evolución y pronóstico del paciente, así como el tiempo hospitalario y las posibles secuelas permanentes (3).

La glucosa es la principal fuente de energía del sistema nervioso central (SNC), ingresa a través de la barrera encefálica, ingresando a la neurona en su mayoría gracias a un transportador específico y solo un $4 \%$ dependiendo de la gradiente de concentración (4). EI SNC no tiene la capacidad de almacenar oxigeno, la tasa metabólica cerebral de la glucosa (TMCG) en un adulto normal es de 0,325 umol/g/min $(2,5,6)$.

Mas del $90 \%$ de la energía del cerebro se obtiene del metabolismo aeróbico de la glucosa por la vía glicolítica, obteniendo 38 ATP por molécula de glucosa, en ayuno prolongado la glicólisis anaeróbica representa menos del $10 \%$, obteniendo 2 moléculas de ATP por molécula de glucosa. Un incremento del metabolismo de la glucosa genera un aumento del consumo de oxigeno; esto da cuenta de la significancia del metabolismo aeróbico en la actividad cerebral (7). La tasa metabólica del oxigeno (TMCO2) en un adulto joven es de $3,4 \mathrm{ml}$ $\mathrm{O} 2 / 100 \mathrm{~g} / \mathrm{min}$ con un rango normal de 1,8 a $3,9 \mathrm{ml} / 100 \mathrm{~g} / \mathrm{min}$ sin que se produzcan alteraciones neurológicas, evidenciándose mínimos cambios durante la actividad mental $(8,9)$.

Algunos investigadores aseguran que la disminución global y regional del flujo sanguíneo cerebral durante la fase aguda de la injuria condiciona una disrupción del metabolismo normal de la glucosa, oxigeno y de las proteínas en especial del metabolismo aeróbico de la glucosa, otros sugieren que hay disturbios en la capacidad oxidativa de la mitocondria cerebral $(6,10)$. La respuesta al trauma craneal se caracteriza por una hiperglicemia con una resistencia periférica a la insulina $(11,12)$.

\footnotetext{
${ }^{1}$ Facultad de Medicina Universidad Nacional de Trujillo. Médico Cirujano

${ }^{2}$ Hospital Regional Docente de Trujillo. Médico Neurocirujano.
} 
La determinación del valor predictivo de la hiperglicemia en la mortalidad de los pacientes con TEC puede ser de mucha utilidad, al tomarlo como un índice precoz, del pronostico malo y de una tórpida evolución con elevada incidencia de complicaciones, serviría de herramienta de triaje para establecer que grupo de pacientes se verían beneficiados con su ingreso a una Unidad de Cuidados Intensivos donde recibirían todo el soporte para un manejo adecuado y una agresiva terapia de neuroprotección, buscando mejorar la relación costo beneficio. Esto motivó a plantearnos siguiente problema ¿Cuál es el valor predictivo de la hiperglicemia en la mortalidad de los pacientes con TEC?, teniendo como objetivo: Determinar si la hiperglicemia tiene valor predictivo significativo en la mortalidad de los pacientes con TEC.

\section{MATERIAL Y METODOS}

El estudio retrospectivo, transversal, de una sola casilla y observacional tuvo como universo a la totalidad de pacientes que ingresaron por el Servicio de Emergencia del Hospital Regional Docente de Trujillo (HRDT) con diagnóstico de TEC entre los meses de Enero a Diciembre del año 2003. y cumplieron los siguientes criterios de selección:

\section{Criterios de inclusión:}

Pacientes que consignen en su historia clínica resultados de glicemia dentro de las primeras 24 horas de ingreso.

\section{Criterios de exclusión:}

Pacientes con antecedentes de: Diabetes Mellitas, politraumatizados, uso de B-bloqueadores, Accidente Cerebro Vascular menor de 6 meses de evolución, con historias clínicas incompletas.

\section{Tamaño de la muestra:}

La muestra estuvo constituida por 164 pacientes con diagnóstico de TEC, para calcular el tamaño de la muestra se utilizó la siguiente formula:

$$
n=\frac{\left(Z_{\text {a/2 }}\right)^{2} p(1-p)}{E^{2}}
$$

donde:

a:Posibilidad de cometer error tipo I p:Tasa anual de fallecidos por TEC (estadística del HRDT-2001)
Z:Valor de la distribución normal asociado a un tipo de error

$\mathrm{E}$ :Error de muestreo

Asumiendo un nivel de confianza del $95 \% \quad\left(Z_{\alpha / 2}=1,96\right)$, tomando un dato estadístico del HRDT con un valor de p: 0,122 (tasa anual de fallecidos con TEC); y con un error de muestreo del $5 \%(E=0,05)$, se calculo que nuestra muestra fue de 164

\section{Método de selección de unidades de muestreo:}

Se empleó el método aleatorio simple para seleccionar los elementos del universo que pasaron a constituir la muestra, para lo cual se uso la tabla de números aleatorios (Tabla de Tippet); siendo nuestra unidad de análisis y unidad de muestreo los pacientes con diagnóstico de TEC, que cumplieron los criterios de inclusión y exclusión; y que consignaron datos completos.

\section{Definición Operacional}

Hiperglicemia: en el presente estudio se utilizo valores mayores o iguales de $160 \mathrm{mg} / \mathrm{dL}$ como punto de corte y así excluir los niveles de la hiperglicemia post trauma (13).

\section{RESULTADOS}

En cuanto a la distribución de pacientes con diagnóstico de TEC según el grado de Escala de Coma de Glasgow (ECG) 17 presentaron TEC Grave de los cuales el $82,4 \%$ presento hiperglicemia; 26 presentaron TEC Moderado de los cuales el $38,4 \%$ pre- sento hiperglicemia; y 121 presentaron TEC Leve de los cuales solo 1 paciente $(0,8 \%)$ presento hiperglicemia. Todo esto evidencia una correlación altamente significativa $(p<0.5)$ entre la hiperglicemia y el grado de severidad del TEC (Tabla $N^{\circ} 01$ ).

Con respecto a la mortalidad de los pacientes con TEC se encontró una tasa de mortalidad del 17,1\% (28 pacientes), de los cuales el 82,1\% (25 pacientes) presentaron hiperglicemia; mientras que en los pacientes que no fallecieron solo el 1,4\% (2 pacientes) presentó hiperglicemia. Nótese que la mortalidad es mayor en los pacientes que presentan hiperglicemia, evidenciándose una asociación altamente significativa entre estas 2 variables en estudio; obteniéndose un valor predictivo positivo de 0,92 y un valor predictivo negativo de 0,96 (Tabla $\mathrm{N}^{\circ}$ 02).

El nivel promedio de glicemia en los pacientes fallecidos fue de $173,4 \mathrm{mg} /$ $\mathrm{dL}$, mientras que en los no fallecidos fue de 95,22 mg/dL, evidenciándose una diferencia estadísticamente muy significativa entre los promedios de glicemia y la mortalidad de los pacientes con TEC (Tabla No 03).

Los niveles promedios de glicemia según el grado de ECG fueron: de $176,24 \mathrm{mg} / \mathrm{dL}$ en TEC grave, 148,92 $\mathrm{mg} / \mathrm{dL}$ en TEC moderado y $90,54 \mathrm{mg} /$ $\mathrm{dL}$ en TEC leve; todo esto demuestra una diferencia estadística muy significativa entre los niveles promedio de glicemia y los grados de TEC según ECG (Tabla No 4).

TABLA Nº 01: Distribución de pacientes con diagnóstico de TEC según grado de ECG tomando como corte glicemia $>160 \mathrm{mg} / \mathrm{dL}$.

\begin{tabular}{|l|l|l|l|l|}
\hline NIVEL DE & \multicolumn{3}{|c|}{ GRADOS DE ECG } & \multirow{2}{*}{ T O T A L } \\
\hline GLICEMIA & \multicolumn{1}{|c|}{ LEVE } & \multicolumn{1}{|c|}{ MODERADO } & GRAVE & \\
\hline GLICEMIA $<160$ & 120 & 16 & 3 & 139 \\
\hline GLICEMIA $>160$ & 1 & 10 & 14 & 25 \\
\hline T O T A L & 121 & 26 & 17 & 164 \\
\hline
\end{tabular}

Chi cuadrado: $89,57 \quad p=0,1 E-12$

TABLA N $N^{\circ}$ 02: Distribución de pacientes con diagnóstico de TEC según mortalidad tomando como corte glicemia $>160 \mathrm{mg} / \mathrm{dL}$.

\begin{tabular}{|l|c|c|c|}
\hline \multirow{2}{*}{$\begin{array}{c}\text { NIVEL DE } \\
\text { GLICEMIA }\end{array}$} & SI & \multirow{2}{*}{ T O T A L } \\
\cline { 2 - 4 } & 5 & NO & 139 \\
\hline$<160$ & 23 & 134 & 25 \\
\hline$>160$ & 28 & 136 & 164 \\
\hline T O T A L & & 2 & \\
\hline
\end{tabular}

Chi cuadrado: $110.8 p=0,1 \mathrm{E}-14$ 
TABLA N ${ }^{\circ}$ 03: Distribución de pacientes con diagnostico de TEC fallecidos y no fallecidos según promedio de glicemia.

\begin{tabular}{|l|l|l|l|l|l|}
\hline & \multicolumn{2}{|c|}{$\begin{array}{c}\text { NIVEL DE GLICEMIA } \\
\text { (mg/dL) }\end{array}$} & \multicolumn{2}{c|}{ VALOR } & \multirow{2}{*}{ SIGNIFICANCIA } \\
\hline & \multicolumn{1}{|c|}{ Promedio } & \multicolumn{1}{|c|}{ D.S. } & $\mathbf{t}$ & $\mathbf{p}$ & \\
\hline FALLECIDOS & 173,4 & 16,9 & & & \\
\cline { 1 - 4 } NO FALLECIDOS & 95,22 & 20,75 & 18,68 & $1,00 E-08$ & Muy significativa \\
\hline
\end{tabular}

TABLA N ${ }^{\circ}$ 04: Distribución de pacientes con diagnostico de TEC con diversos grados de ECG según promedio de glicemia.

\begin{tabular}{|l|l|l|l|l|l|}
\hline \multirow{2}{*}{$\begin{array}{l}\text { GRADO DE } \\
\text { ECG }\end{array}$} & \multicolumn{2}{|c|}{$\begin{array}{c}\text { NIVEL DE GLICEMIA } \\
\text { (mg/dL) }\end{array}$} & \multicolumn{2}{c|}{ VALOR } & \multirow{2}{*}{ SIGNIFICANCIA } \\
\cline { 2 - 5 } & \multicolumn{1}{|c|}{ Promedio } & \multicolumn{1}{c|}{ D.S. } & $\mathbf{t}$ & $\mathbf{p}$ & \\
\hline GRAVE & 176,24 & 11,82 & & & \\
\hline MODERADO & 148,92 & 24,3 & 5,38 & $2,00 \mathrm{E}-07$ & \multirow{2}{*}{$\begin{array}{l}\text { Muy significativa } \\
\text { Muy significativa }\end{array}$} \\
\hline LEVE & 90,54 & 16,6 & 20,51 & $1,00 \mathrm{E}-08$ & \\
\hline
\end{tabular}

\section{DISCUSION}

Los grados de TEC según la EGC en relación a la hiperglicemia tomando como punto de corte el valor de glicemia $>160 \mathrm{mg} / \mathrm{dL}$, evidencia que los pacientes con TEC grave presentan en un mayor porcentaje $(82,35 \%)$ hiperglicemia en contraposición a los pacientes que presentan TEC leve quienes mayormente $(99,17 \%)$ no presentan hiperglicemia, esto asociado a una alta significancia estadística $(p<0.01)$ demuestra que hay una correlación entre ambas variables. Según Yong y col. en 1989; en estudios realizados en pacientes con TEC severos se observó que en la respuesta al estrés se incluía a la hiperglicemia, la cual era más significativa, conforme la ECG era menor (2); este dato fue corroborado por Lam A.M. y col. en 1991, donde se encontró que esta relación era mayor, y a un alto valor de glicemia encuentra un menor valor en la ECG (3). Esto se correlaciona con los estudios de Lazar L. y col en 1997, quien demostró que los pacientes con TEC presentan mayor hiperglicemia que los que no presentaron TEC (1).

Revisando si la hiperglicemia tiene o no una relación con la mortalidad en los pacientes con TEC, se evidencia una elevada probabilidad de sufrir este evento, expresado por un OR de 308,2 y una $p$ significativa $(p<0,1$ x10-14), En Perú Espinoza JM, 1998 encontró que la hiperglicemia tiene un valor predictivo para mortalidad de pacientes con TEC de 0,87; con una p significativa y con una sensibilidad de $69 \%$ y una especificidad del $87 \%$ (7)(14).

Al correlacionar el promedio de glicemia entre los pacientes fallecidos y los no fallecidos se evidencio una asociación estadísticamente muy sig- nificativa con un $p<0.01$, de ello podemos afirmar que los pacientes que presentan hiperglicemia tienen mayor riesgo de fallecer, esto debido principalmente a que la hiperglicemia se correlaciona con una disminución progresiva de la TMC O2.(1) (14) (15). En el presente estudio se evidencia que existe una asociación estadística muy significativa entre los niveles promedios de glicemia y los grados de TEC según la ECG con un $p<0.01$ siendo mayor el promedio de glicemia en el TEC grave y menor en el TEC leve. Desai D. y col en 1989, encuentra que el incremento de la glucosa empeoraba la evolución de los pacientes con TEC y esto se reflejaba en el descenso de la Escala de Coma Glasgow (6).

El peso del cerebro representa el 1 a$2 \%$ del peso corporal, recibe aproximadamente el $20 \%$ del gasto cardiaco, consume el $40 \%$ del oxigeno y utiliza el $25 \%$ de la glucosa suministrada, cerca del $45 \%$ de las energía se utiliza en mantener la integridad celular y $55 \%$ en preservar la función neuronal (16)..En el trauma la TMCO2, se reduce a niveles de 0,6 a $1,2 \mathrm{umol} / \mathrm{g} / \mathrm{min}$. La reducción de la TMCO2 se realiza en forma proporcional al grado de severidad del trauma $(1,17)$. Un trauma craneal acompañado de hemorragia y de hipotensión compromete más aun la tasa metabólica de $\mathrm{O} 2$, pacientes que presentan una ECG $<8$ tendrán un descenso en la $\mathrm{TMCO} 2$ de un $50 \%$; por otro lado, existen estudios en los que se sugieren una relación fuerte entre el flujo sanguíneo cerebral y la tasa metabólica cerebral de oxigeno sin cambios en la diferencia arteriovenosa de oxigeno que son consistentes con una disminución de los requerimientos metabólicos cerebrales (2).Duckrow R.B. en 1995, encontró que el flujo sanguíneo cerebral dismi- nuía durante la hiperglicemia, pero no identificaba los mecanismos por lo que se producía esto (10).

Michaud L. J. y col en donde concluye que la hiperglicemia que se presenta en los pacientes con TEC, agrava el daño por isquemia y empeora la evolución de estos pacientes (5). Cherian L,1997, identifico que la hiperglicemia aumentaba el daño producido por la isquemia y por consiguiente se producía un mayor deterioro en los niveles de conciencia del paciente, esto se agrava aún más cuando el paciente recibe soluciones glucosadas en comparación con las soluciones salinas (8). Lo cual fue demostrado por Yura y col 1991 (9).

La contribución de nuestro trabajo radica en la utilización de la glicemia como herramienta de triaje para valorar el pronostico y la severidad de los pacientes con TEC al ingreso a emergencia; y este parámetro asociado a la ECG nos permitirán la detección precoz de los pacientes que tienen alta probabilidad de fallecer por TEC. Por todo ello recomendamos incorporar a la glicemia en el protocolo de atención al paciente con TEC en los diversos Servicios de Emergencia de los establecimientos de salud de nuestro país.

\section{CONCLUSION}

La hiperglicemia, mayor de 160mg/ $\mathrm{dL}$, se asocia significativamente con la mortalidad de los pacientes con Traumatismo Cráneo Encefálico; con un valor predictivo positivo de 0,92 y un valor predictivo negativo de 0,96 . 


\section{REFERENCIAS BIBLIOGRÁFICAS}

1. Lazar L, Erez I, Gutermacher M, Katz SM. Brain concussion produces transient hypokalemia in children. J Pediatric Surg 1997; 32(1):88-90.

2. Yong B, Ott L, Dempsey R, Haack D, Wicghi HD. Relationship between admission hyperglycemia and neurologic outcome of severly brain injured patients. Ann Surg 1989; 210(4):466-72.

3. Lam AM, Winn HR, Cullen BF, Owen T. Hiperglycemia and neurological outcome in patients with head injury. J Neurosurg 1991; 75(4):545-51.

4. Aswal S, Schneider S, Tomasi L. Hyperglycemia during physical stress. Am J Med 1996; 40(5):374-8.

5. Michaud LJ, Rivara FP. Elevated initial blood glucose levels and poor outcome following severe brain injuries. in Neurosurgical Intensive Care. Neurosurg Clin N Am 2000; 9(4):145-9.

6. Desai D, March R, Watters JM. Hyperglycemia after trauma increases with age. J Trauma 1989; 29(6):719-23.

7. Espinoza JM. Valor predictivo de la hiperglicemia en el pronostico y evolución de pacientes con TEC. Tesis para optar el grado de Especialista en Medicina Intensiva. Lima: Universidad Nacional Mayor de San Marcos; 1998.

8. Cherian L, Goodman JC, Robertson C. Hyperglycemia increases brain injury caused by secondary ischemia after cortical impact injuryin rats. Crit Care Med 1997; 25(8):1378-83.

9. Yura D, Yura S. Effects of hyperglycemia on ischemic brain damage, local cerebral blood flow and ischemic cerebral edema. Hokkaido Igaku Zasshi 1991; 15(2):1-15.

10. Ducrow RB. Decreased cerebral blood flow during acute hyperglycemia. Brain Res 1995; 562:145-50.

11. Neil AR. Cerebral Blood flow measurement in Neurosurgical Intensive Care. Neurosurg Clin N Am 1994; 5(4):98590.

12. Ritter A, Marie E. Cerebral Metabolism. Neurosurg Clin N Am 1994; 5(4):842-9.

13. Revelle RK. Trauma severety indices. Ann of Emerg 2000; 36:378-86.

14. Parish RA, Webb KS. Hyperglycemia is not poor prognostic sig in head injured children. J Trauma 1988; 28 (4):517-9.

15. Pulseinelli W, Levy D. Regional cerebral blood flow and glucose metabolism following transiet forebrain isquemia. Ann Neurol 1988; 499(11):352-8.

16. Goodman J, Narayan MD, Contant F, Grossman G. The effect of glucose administration on carbohydrate metabolism after head injury. J Neurosurg 1991; 74:43-50.

17. Bouma GJ, Muizelaar JP, Choi SC, Newlon PG, Young HF. Cerebral circulation and metabolism after severe traumatic brain injury: The elusive role of ischemia. J Neurosurg 1991; 75:685-93.

18. Mormomtoy W. Elaboración del protocolo de Investigación. 2a ed. Lima: Ed. Universidad Peruana Cayetano Heredia; 1994.

Email: cequisa@terra.com 